\title{
SYNTHESIS OF HIERARCHICAL ZSM-5 BY SILANIZATION AND ALKOXYLATION OF PROTOZEOLITIC UNITS
}

\author{
D. P. Serrano ${ }^{1,2^{*}}$, J. Aguado ${ }^{3}$, J. M. Escola 3 , A. Peral ${ }^{1}$, G. Morales ${ }^{3}$, E. Abella ${ }^{3}$
}

\section{Department of Chemical and Energy Technology, ESCET}

Rey Juan Carlos University, Móstoles, 28933, Spain

2IMDEA Energy Institute, Móstoles, 28933, Spain

3Department of Chemical and Environmental Technology, ESCET

Rey Juan Carlos University, Móstoles, 28933, Spain

Published on:

Catalysis Today, 168 (2011) 86-95,

doi:10.1016/i.cattod.2010.12.040

*to whom correspondence should be addressed

Tel: +34 914887450

Fax: +34914887068

e-mail: david.serrano@urjc.es 


\section{Abstract}

Hierarchical ZSM-5 zeolite has been synthesized by means of a method involving a precrystallization stage to form the protozeolitic units, the addition and subsequent grafting of both silanization and alkoxylation agents, and a final hydrothermal crystallization. The influence of the alkoxylation with different alcohols (methanol, ethanol, 2-propanol and n-butanol) on the properties of the final hierarchical ZSM-5 samples has been investigated. In every case, the alcohol addition increased the incorporation of the seed silanization agent as it decreases the gel viscosity. In addition, the presence of alcohols deeply affects the physicochemical properties of the final materials. The samples prepared with 2-propanol and methanol were highly crystalline and presented improved textural properties with regard to the reference h-ZSM-5 and n-ZSM-5. In contrast, the samples obtained with ethanol and n-butanol were partially and totally X-ray amorphous, respectively. ${ }^{1} \mathrm{H}$ and ${ }^{13} \mathrm{C}-\mathrm{CP}$ solid state MAS NMR spectra proved the alkoxylation of the external surface of the protozeolitic units. Catalytic cracking of LDPE pointed out the higher TOF values obtained over the hierarchical samples prepared with methanol and 2-propanol due to a right combination of accessibility and crystallinity in these materials. The differences observed among the samples prepared with alcohols were ascribed to the strong interaction produced between the silanization agent and the linear alcohols on the surface of the protozeolitic nanounits, which form a very stable protective layer, hindering their aggregation and subsequent crystallization.

Keywords: hierarchical zeolite, ZSM-5, silanization, alkoxylation 


\section{Introduction}

Hierarchical zeolites are characterized by the presence of a bimodal porous structure, containing both micropores and mesopores/macropores. These materials are currently a subject of great interest for the scientific community worldwide due to their remarkable properties [1-10]. These include chiefly improved diffusion, reducing mass transfer constraints [11-13], resistance to deactivation $[14,15]$, better possibilities for surface functionalization [16] and improved dispersion of other active phases like metals and metal oxides [17]. Besides the classical methods for generating mesoporosity (steam treatment [18], acid treatment [19]), new synthetic procedures have been developed in the last years such as carbon templating [4], desilication [20] and silanization based methods (seed silanization [3], polymer silanization [10] and amphiphile silanization [9]). These methods allow broadening the possible applications of the materials so obtained since they lead to the development of secondary porosities having different pore sizes and contributions to the overall textural properties of the zeolite. Thus, the carbon templating route usually leads towards the preparation of MFI zeolite with mesopores with a mean size of 10 $\mathrm{nm}$ while for instance, the mesopores generated by polymer silanization are around $2.0-3.0 \mathrm{~nm}$. In addition, the BET surface area of the materials varies within $350-785 \mathrm{~m}^{2} \mathrm{~g}^{-1}$ depending on the synthetic procedure, the larger values being attained with the ZSM- 5 zeolite prepared by the seed silanization method [21]. Many of these procedures can be extended to other zeolites besides ZSM-5, such as mordenite [22], Beta [23], etc. Additionally, several recent literature reviews can be found dealing with the topic of hierarchical zeolites [24, 25].

In previous works, our group developed a procedure for the preparation of hierarchical zeolites, named "seed silanization", using relatively bulky organosilanes which are grafted onto the external surface of protozeolitic nuclei $[3,21-23,26-28]$, hindering partially their aggregation into bigger entities and, therefore, avoiding the growth of conventional zeolite crystals. After calcination, the space initially occupied by the organosilane is released giving rise to the 
formation of mesopores. Accordingly, the seed silanization method comprises the following four stages: a) precrystallization of the zeolite synthesis gel to promote the formation of protozeolitic nanoparticles; b) functionalization of the protozeolitic units by reaction with the organosilanes; c) crystallization of the functionalized protozeolitc units under standard hydrothermal conditions; d) calcination to remove both the silanization agent and the zeolite structure directing agent. In the case of ZSM-5, the product so obtained is made up of $200-400 \mathrm{~nm}$ aggregates formed by ultrasmall ZSM-5 nanounits $(5-20 \mathrm{~nm})$, having a high proportion of mesopores among them. The presence of this mesoporosity involves a high concentration of hydroxyl groups on the mesopore surface, which increases the hydrophilic properties of the zeolites prepared by seed silanization [26].

The mesopore size and the nanounit size depend on the used organosilane. The best results were attained with phenylaminopropyltrimethoxysilane (PHAPTMS), leading towards materials having $2.0-5.0 \mathrm{~nm}$ mesopores and pore walls formed by the smallest ZSM-5 nanounits $(5-10 \mathrm{~nm})$ [27]. Moreover, TEM images showed clearly that adjacent crystalline nanounits present the same orientation of the crystallographic planes [27], having a high degree of intergrowth, which gives rise to larger crystalline domains and improves their stability. It was also concluded that most of the aluminium was incorporated into tetrahedral positions of the hierarchical zeolite framework, as determined by ${ }^{27 A l-M A S ~ N M R ~ a n a l y s e s ~[3] . ~ A d d i t i o n a l l y, ~ 1 D ~}$ and 2D NMR measurements indicated that the silanization agent is located grafted over the external surface of the zeolitic nanounits [21].

The properties of the hierarchical zeolites obtained by the seed silanization treatment depend not only on the nature of the seed silanization agent but also on its concentration and on the conditions employed during the precrystallization stage. Thus, an optimum of $12 \mathrm{~mol} \%$, referred to the total silica content, was found with phenylaminopropyltrimethoxysilane as seed silanization agent. On the other hand, the precrystallization step for the formation of the 
nanozeolitic precursors containing $\mathrm{TPA}^{+}$occluded is completely necessary, leading to an amorphous material in its absence [3]. In addition, lower temperatures $\left(40^{\circ} \mathrm{C}\right)$ in the precrystallization step gave rise to the highest BET surface area so far reported for a hierarchical ZSM-5: $785 \mathrm{~m}^{2} \mathrm{~g}^{-1}$ [21]. Likewise, it must be pointed out that the seed silanization strategy can be considered as a general method for synthesizing zeolites with hierarchical porosity as it has been successfully applied to a variety of zeolites aside of ZSM-5, such as ZSM-11 [3], Beta [23], mordenite [22] and TS-1 [24].

The goal of the present work is to improve and to optimize the seed silanization method. We have found that when a high proportion of organosilane is introduced into the synthesis medium (> $10 \mathrm{~mol} \%$, relative to the total silica content of the starting gel) a highly viscous gel is formed. This fact hinders the diffusion of the organosilane in the gel and its anchoring onto the zeolitic seeds as well as it promotes the appearance of homocondensation reactions between organosilane molecules, leading to lower quality materials. One possibility to improve the silanization reaction is to dilute the silanization agent by the addition of solvents. Likewise, the role played by the alcohol deserve deep study since it is known that alcohols influence the synthesis of zeolites, e.g. reducing the aggregation of nanoparticles and leading to smaller nanoparticles sizes [26]. In the present work, a variety of alcohols have been added during the silanization step in the synthesis of hierarchical ZSM-5 zeolite in order to enhance the effect of the silanization agent on the physicochemical and catalytic properties of this class of materials.

\section{Experimental}

\subsection{Material synthesis}

A solution containing the ZSM-5 protozeolitic units was prepared with the following molar composition: $1 \mathrm{Al}_{2} \mathrm{O}_{3}: 60 \mathrm{SiO}_{2}$ : $11 \mathrm{TPAOH}$ : $1500 \mathrm{H}_{2} \mathrm{O}$. Tetraethoxysilane (TEOS, 98\%; Aldrich), tetrapropylammonium hydroxide (TPAOH, 40\%; Alfa), aluminum isopropoxide (AIP; Aldrich), and 
distilled water were used as starting materials. The clear precursor solutions were precrystallized under reflux with stirring $(100 \mathrm{rpm})$ at $90{ }^{\circ} \mathrm{C}$ for $20 \mathrm{~h}$. The resulting protozeolitic units were then functionalized with phenylaminopropyltrimethoxysilane (PHAPTMS; Aldrich) at $90^{\circ} \mathrm{C}$ for $24 \mathrm{~h}$. The organosilane was incorporated in a proportion of $12 \mathrm{~mol} \%$ with respect to the silica content in the gel. The alcohol was added with the organosilane using an organosilane / alcohol weight ratio of 0.076. The alcohols tested were: methanol, ethanol, 2-propanol and n-butanol. After this stage, the major part of the alcohol was removed from the synthesis medium by means of a rotary evaporator. For the alcohol removal, controlled vacuum and a silicone bath at temperatures in the range $50-70^{\circ} \mathrm{C}$ (depending on the type of alcohol to be removed) were employed. The solvent distilled from the synthesis medium was collected in a condensate-collecting round bottom flask. Additionally, a glass trap refrigerated with liquid nitrogen was located between the evaporator flask and the vacuum pump in order to collect the solvent evaporated but not accumulated in the condensate flask. About 30 minutes were enough to remove a liquid amount similar to that of the alcohol added after the precrystallization step to the synthesis medium. The composition of the collected liquid phase was determined by means of gas chromatography, which allowed the total quantity of evaporated alcohol to be calculated. Finally, crystallization was carried out in Teflon ${ }^{\circledR}$ lined stainless-steel autoclaves under static conditions and autogenous pressure at $170^{\circ} \mathrm{C}$ for 7 days. The solid products obtained were separated by centrifugation, washed several times with distilled water, dried overnight at $110^{\circ} \mathrm{C}$ and calcined in air at $550^{\circ} \mathrm{C}$ for $5 \mathrm{~h}$. The synthesized samples were named as h-ZSM-5 (A), wherein A stands for the employed alcohol: methanol $(A=$ meth), ethanol ( $A=$ eth), 2-propanol ( $A=2-p r o p)$, and n-butanol $(A=n-b u t)$.

Additionally, two reference samples were prepared for comparison. Firstly, a hierarchical ZSM-5 zeolite, synthesized following an identical procedure but omitting the addition of any alcohol, and secondly, a reference nanocrystalline ZSM-5 zeolite prepared following the same methodology and conditions but omitting the addition of both alcohols and PHAPTMS. The 
hierarchical reference sample was denoted as $\mathrm{h}-\mathrm{ZSM}-5$ and the nanocrystalline MFI reference as n-ZSM-5.

\subsection{Characterization of the materials}

Powder X-ray diffraction (XRD) patterns were measured using a Philips X'PERT MPD diffractometer (Cu $K_{\mathrm{a}}$ radiation) with step size and counting time of $0.02^{\circ}$ and $10 \mathrm{~s}$, respectively. Fourier transform infrared (FTIR) spectra were recorded in a Mattson Infinity Series spectrophotometer with a resolution of $4 \mathrm{~cm}^{-1}$ using the $\mathrm{KBr}$ pellet technique. Nitrogen adsorptiondesorption isotherms at $77 \mathrm{~K}$ were obtained in a Micromeritics ASAP 2010 instrument, whereas argon adsorption-desorption isotherms at $87 \mathrm{~K}$ were acquired using a Quantachrome Autosorb 1 MP automated gas sorption system. In both cases, the analyses were carried out after outgassing the samples at $300^{\circ} \mathrm{C}$ under vacuum for $16 \mathrm{~h}$. BET surface area calculations were performed using the relative pressure range of 0.05 to 0.16 .

The Si/Al atomic ratios of the samples were determined by inductively coupled plasma spectroscopy (ICP) with a VARIAN VISTA AX apparatus, while elemental chemical analyses for $\mathrm{C}, \mathrm{N}$ and $\mathrm{H}$ were carried out using a Vario EL III instrument. Thermogravimetric (TGA) analyses were performed using a SDT 2960 instrument. Transmission electron microscopy (TEM) images were obtained with a JEOL 2000 electron microscope operating at $200 \mathrm{kV}$.

Solid-state ${ }^{1} \mathrm{H}$ and ${ }^{13} \mathrm{C}$ NMR experiments were conducted at room temperature with a magnetic field strength of $9.4 \mathrm{~T}$ on a high-resolution VARIAN INFINITY PLUS 400 NMR spectrometer operating at frequencies of $400 \mathrm{MHz}$ for ${ }^{1} \mathrm{H}$ and $100.40 \mathrm{MHz}$ for ${ }^{13} \mathrm{C}$. Proton and carbon chemical shifts were referenced to the corresponding nuclei in tetramethylsilane. Magicangle spinning (MAS) NMR spectra were obtained using a $7 \mathrm{~mm}$ double-resonance broadband MAS probehead with a spinning rate of $6 \mathrm{kHz}$. Solid-state single-pulse ${ }^{1} \mathrm{H}$ MAS NMR spectra 
were recorded using a $1.7 \mu \mathrm{s} \pi / 2$ pulse, a recycle delay of $20 \mathrm{~s}$ and 32 transients. Solid-state ${ }^{13} \mathrm{C}$ CP-MAS NMR spectra were recorded with a ${ }^{1} \mathrm{H} \pi / 2$ pulse of $6 \mu \mathrm{s}$, followed by a $5 \mathrm{~ms} \mathrm{CP}$ contact time, a recycle delay of $2 \mathrm{~s}$, and 3200 transients.

\subsection{Catalytic tests}

Catalytic properties of the zeolitic samples were tested for the cracking of low-density polyethylene (LDPE). The reactions were carried out in a stainless steel batch reactor provided with a helicoidal stirrer swept by a continuous nitrogen flow $\left(35 \mathrm{~mL} \cdot \mathrm{min}^{-1}\right)$ to ensure that the LDPE conversion takes place under inert conditions and to favor the removal of the volatile products. In a typical experiment, $10 \mathrm{~g}$ of LDPE were introduced into the reactor together with $0.1 \mathrm{~g}$ of the catalyst (LDPE/catalyst mass ratio of 100). The reactions were performed at $340^{\circ} \mathrm{C}$ for $2 \mathrm{~h}$. The volatile products leaving the reactor were separated into gases and liquids by means of an icetrap, their composition being determined by gas chromatography.

\section{Results and Discussion}

Silanization of protozeolitic units has shown in earlier works to be an effective method for developing a hierarchical pore system in zeolites, leading to materials with improved textural properties, enhanced accessibility and high catalytic activity in the processing of bulky compounds. Increasing the amount of silanization agent allows the variation of those properties to be controlled. However, when the amount of silanization agent is over $10 \mathrm{~mol} \%$, regarding the total silica content, the gel becomes highly viscous limiting its interaction with the protozeolitic units and reducing its effect on the hierarchical zeolite properties. In this context, the present work was initially aimed to study the introduction of different alcohols during the seed silanization step to reduce the viscosity of the gel. With this purpose, methanol, ethanol, 2-propanol and n-butanol were selected as alcohols for being introduced into the synthesis gel along with the silanization 
agent. A Cannon-Fenske routine-calibrated viscosimeter was used to measure the viscosity of the alcohol-containing gels. The calculated kinematic viscosities were 7.0, 7.6, 8.3 and $9.1 \mathrm{cSt}$ $\left(\mathrm{mm}^{2} \cdot \mathrm{s}^{-1}\right)$, for the methanol, ethanol, 2-propanol and n-butanol containing solutions, respectively. On the other hand, when no alcohol was added to the synthesis medium the measured kinematic viscosity was $12.1 \mathrm{cSt}\left(\mathrm{mm}^{2} \cdot \mathrm{s}^{-1}\right)$, demonstrating that the addition of alcohols largely diminishes the viscosity of the gel. Additionally, a better solubility of the organosilane seems to be also attained. Both causes are expected to improve the grafting of the silanization agent. After the silanization treatment the alcohols are removed by evaporation, although not completely. The amount of alcohols remaining in the gel solution after the evaporation is in the range $10-18 \%$ of the added one, depending on the chosen alcohol, according to the GC analysis of the liquids collected from the rotary evaporator. Consequently, the subsequent crystallization step occurs in a system with a low concentration of alcohol molecules.

The samples prepared incorporating different alcohols in the silanization step were first studied by elemental analyses. The results obtained pointed out that the content of silanization agent in the samples prepared with alcohols is higher than in the reference h-ZSM-5. When no alcohol is added, the content of PHAPTMS in the zeolite is clearly lower than in the gel, giving $8.8 \%$ compared to $12 \mathrm{~mol} \%$ in the gel. In contrast, when alcohols are added, the content of PHAPTMS in the zeolites surpass that of the gel, especially for the samples $h$-ZSM-5 (2-prop), $h$ ZSM-5 (meth) and h-ZSM-5 (eth), which is around $13.0 \mathrm{~mol} \%$. Consequently, the presence of the alcohol increases the amount of silanization agent anchored over the zeolite, which can be interpreted as a consequence mainly of the reduction in the system viscosity caused by the alcohol during the silanization treatment.

Figures 1.A and B illustrate the XRD patterns and FTIR spectra respectively, of the samples prepared under the presence of different alcohols, as well as those corresponding to two reference ZSM-5 samples: a nanocrystalline ZSM-5 (n-ZSM-5) and a hierarchical ZSM-5 
prepared by the seed silanization recipe but without any alcohol addition. Nanocrystalline ZSM-5 showed clearly the typical reflections of ZSM-5 zeolite. The reference h-ZSM-5 sample displays broader and less intense diffraction peaks, which is indicative of the smaller size of the constituting nanocrystalline domains. Low angle XRD patterns $\left(2 \theta=1-10^{\circ}\right.$, data not shown) discarded the presence of any mesostructured phase in the hierarchical ZSM-5 samples. Additionally, no broad bottom reflection in the range $2 \Theta \sim 20-30^{\circ}$ is appreciated bearing out the absence of any amorphous phase for the h-ZSM- 5 sample. The XRD patterns of the samples prepared with two of the alcohols (methanol and 2-propanol) are rather similar to the reference hierarchical h-ZSM-5 indicating that the addition of these alcohols does not affect the crystallinity of the materials. However, the sample synthesized with n-butanol is completely X-ray amorphous, almost without any trace of reflection from crystalline material. Likewise, the XRD pattern of the $h$ ZSM-5 (eth) showed MFI zeolite peaks with low intensity, whereas the presence of a broad shoulder indicates it contains some amorphous material, although in a reduced proportion compared to the sample prepared with n-butanol. These XRD results were confirmed by means of FTIR measurements. As shown in Figure 1.B, for the n-ZSM-5 sample two characteristic bands are clearly observed: one at $1220 \mathrm{~cm}^{-1}$, typical of well crystallized zeolites and another one at 550 $\mathrm{cm}^{-1}$, assigned to the asymmetric stretching mode of the five-membered rings of ZSM-5 zeolite [29], which is absent in amorphous materials. This band is clearly appreciated at $546 \mathrm{~cm}^{-1}$ for all the samples except for h-ZSM-5 (n-but), wherein it is displaced at higher wavenumber $\left(556 \mathrm{~cm}^{-1}\right)$ and with a much lower intensity. In order to quantify the crystallinity among the different samples, the optical density ratio $(\mathrm{ODR})$, defined as the ratio $\left(\left.\right|_{550} / /_{450}\right)$, was determined (see Table 1$)$. According to literature, a well crystallized zeolite possesses an ODR value above 0.7 [30]. It can be clearly appreciated that all the samples (except h-ZSM-5 (n-but)) exhibited ODR above 0.7, with higher values $(0.8)$ for both reference $n-Z S M-5$ and h-ZSM-5 materials. Even the sample synthesized under the presence of ethanol (h-ZSM-5 (eth)), whose XRD pattern exhibited certain 
amount of amorphous material, showed an ODR value of 0.726 , suggesting it is formed largely by MFI structure units. Only for the h-ZSM-5 (n-but) sample, a far distant ODR value was attained (0.492), confirming the previous XRD data about the amorphous nature of this sample.

These results were unexpected as they showed a strong effect of the type of alcohol on the zeolite crystallinity. It could be argued that this is caused just by the higher degree of silanization agent incorporation onto the protozeolitic units achieved in the presence of alcohols, which may hinder the zeolite crystallization. However, this explanation must be ruled out as samples with a high content of silanization agent exhibit also a good crystallinity, as it is the case of the materials obtained with methanol and isopropanol. The origin of these sharp differences among the samples should be linked to changes in the properties of the protozeolitic units induced by the presence of the alcohols during the silanization step and also, although in a lower amount, during the hydrothermal crystallization.

Textural properties of the hierarchical ZSM-5 samples, determined from the $\mathrm{Ar}$ adsorption-desorption isotherms at $87 \mathrm{~K}$, are shown in Table 1. The crystalline samples prepared with alcohols (methanol, ethanol and 2-propanol) present very high BET surface areas, with values around $700 \mathrm{~m}^{2} \mathrm{~g}^{-1}$, which are clearly higher than those of the two reference samples (420 and $\left.609 \mathrm{~m}^{2} \mathrm{~g}^{-1}\right)$. However, sample h-ZSM-5 (n-but) possesses clearly a lower BET surface area $\left(585 \mathrm{~m}^{2} \mathrm{~g}^{-1}\right)$ than the samples synthesized with the other three alcohols. Table 1 also provides data relative to the micropore surface $\left(S_{Z M P}\right)$, and the secondary porosity surface $\left(S_{S P}\right)$. Seemingly, the secondary porosity of all the samples prepared with alcohols is higher than that of the reference h-ZSM-5, which reflects the influence of the presence of the alcohols in enhancing the hierarchical porosity and improving the textural properties. The development of the secondary porosity is usually at the expense of the zeolite micropores. Hence, the micropore surface area drops from $267 \mathrm{~m}^{2} \mathrm{~g}^{-1}$ for h-ZSM-5 (2-prop) to $28 \mathrm{~m}^{2} \mathrm{~g}^{-1}$ for h-ZSM-5 (n-but). It can be appreciated also that the $\mathrm{S}_{\mathrm{BET}}, \mathrm{S}_{\mathrm{ZMP}}$ and $\mathrm{S}_{\mathrm{SP}}$ of the sample prepared with 2-propanol surpasS 
those of the reference h-ZSM-5. Consequently, this material clearly proves that the addition of 2propanol during silanization improves the textural properties of the hierarchical ZSM-5.

Other aspect of interest is the pore size distribution of the samples, which has been calculated by application of the NLDFT model to the Ar isotherms at $87 \mathrm{~K}$. Figure 2 shows the cumulative pore volume as well as the pore size distribution obtained from the Ar adsorption isotherms at $87 \mathrm{~K}$. The pore size distributions confirm that most samples show a hierarchical porosity and reveals significant differences among them. The steps observed in the cumulative pore volume curves correspond with different levels of porosity. The reference n-ZSM-5 is a conventional zeolite having just the typical MFI micropores. H-ZSM-5 (2-prop) presented mesopores slightly higher $(2.0-6.0 \mathrm{~nm})$ than those of the reference h-ZSM-5 $(2.0-4.0 \mathrm{~nm})$, while the sample h-ZSM-5 (meth) shows mesopores centered at 2.0 and $7.0 \mathrm{~nm}$. In contrast, samples h-ZSM-5 (eth) and h-ZSM-5 (n-but) showed quite broader pore size distributions, with mesopores between 2.0 and $60.0 \mathrm{~nm}$. On the other hand, for samples h-ZSM-5, h-ZSM-5 (2prop) and h-ZSM-5 (meth) the contribution of the microporosity is attenuated, whereas sample $h$ ZSM-5 (n-but) does not contain micropores. Especially interesting is the case of the h-ZSM-5 (eth) sample as it exhibits up to three levels of porosity: zeolitic micropores, medium size mesopores (2 - $3 \mathrm{~nm})$ and large mesopores $(10-40 \mathrm{~nm})$. These results are consistent with this sample being formed by a mixture of amorphous and crystalline phases as above concluded from the XRD pattern.

The samples have been also investigated by TG/DTG analyses (see Figure 3). The total weight losses of the samples prepared by the seed silanization method, including the reference $h$ ZSM-5, show practically the same total weight loss, within the $25-27 \%$ range. However, the TG and DTG of the hierarchical samples prepared with and without alcohols display some differences. Figure 3.A) exhibits the TG and the derivative curve (DTG) corresponding to the assynthesized n-ZSM-5 sample, wherein a main peak at $463^{\circ} \mathrm{C}$ corresponding to the decomposition 
of the TPA ${ }^{+}$cations, strongly retained inside the micropores, is appreciated. Figure 3.B) displays the TG and DTG corresponding to the as-synthesized h-ZSM-5, obtained using a seed silanization approach but without alcohols. As inferred from the graph, three peaks of weight loss can be appreciated in the DTG of this sample, centered at 285,461 and $563^{\circ} \mathrm{C}$, respectively. The second one is again related to the TPA ${ }^{+}$occluded within the zeolite micropores. The peak at $285^{\circ} \mathrm{C}$ is assigned mostly to the fragmentation and combustion of the seed silanization agent (PHAPTMS), while the one at $568^{\circ} \mathrm{C}$ may be related to both the combustion of carbonaceous residues formed from the decomposition of organic compounds and surface dehydroxylation processes. Figure 3.C) shows the TG and DTG of as-synthesized hierarchical ZSM-5 (eth) sample. The aforementioned peaks are also present in the TG/DTG of this sample, along with an additional small peak at $200^{\circ} \mathrm{C}$. This peak represents a small share in the total weight loss $(1-2 \%)$ and it could denote the possible presence of ethoxy moieties $\left(\mathrm{CH}_{3} \mathrm{CH}_{2} \mathrm{O}-\right)$ anchored over the zeolite outer surface. A quite similar peak is also observed in the case of the sample prepared adding n-butanol with the silanization agent. This fact suggests that the role of the alcohols is not just to act as solvents during the silanization treatment but they may undergo alkoxylation reactions, being anchored to the surface of the protozeolitic units, which could explain their strong effect on the crystallinity and properties of the materials finally obtained.

TEM micrographs of the reference samples (n-ZSM-5 and h-ZSM-5) are shown in Figure 4. The $\mathrm{n}-\mathrm{ZSM}-5$ sample is made up of aggregates of $30-70 \mathrm{~nm}$ nanocrystals. In contrast, $\mathrm{h}$ ZSM-5 is formed by $200-400 \mathrm{~nm}$ globular aggregates formed by quite smaller nanounits $(5-10$ $\mathrm{nm})$. Figure 5 illustrates the micrographs of the hierarchical ZSM- 5 samples prepared with alcohol addition in the silanization step. Both h-ZSM-5 (2-prop) and h-ZSM-5 (meth) exhibit an appearance very similar to that of the reference h-ZSM-5. Hence, globular agglomerates around $200-400 \mathrm{~nm}$ formed by small nanounits are clearly visible. The presence of diffraction fringes confirms that these materials are crystalline in spite of being formed by ultra-small nanounits. The 
latter are not really independent but they show a high degree of intergrowth, as indicated by the diffraction lines having the same orientation over adjacent nanounits. On the other hand, the micrographs of h-ZSM-5 (eth) did not show the presence of the globular agglomerates. Instead, a micrometer size matrix is observed containing some dark particles embedded, which are presumably the precursors of the future globular agglomerates. This finding confirms that this samples is really a mixture of amorphous (the matrix) and crystalline (the dark particles) phases. Finally, neither globular agglomerates nor crystalline material are appreciated in the micrographs of h-ZSM-5 (n-but), confirming the previous data from XRD and FTIR. This sample is formed by nanounits having a low degree of aggregation. These results are very interesting since they indicate the existence of a direct relationship between the aggregation degree and the appearance of crystalline features in this system. The materials obtained using ethanol or $n$ butanol in the silanization step contain an amorphous phase, which is formed by nanounits with little aggregation, suggesting that these alcohols have largely avoided the growth of the globular aggregates. This is probably related to the above proposed alkoxylation of the protozeolitic nanounits, which may reinforce the role of the silanization agent in hindering the zeolite crystal growth by an aggregative mechanism. However, it is still unclear why this effect is observed with ethanol and n-butanol and not when using methanol or 2-propanol as solvents during the seed silanization step.

Solid-state ${ }^{1} \mathrm{H}$ MAS NMR room-temperature measurements of the as-synthesized samples were carried out, the corresponding spectra being shown in Figure 6 . In the absence of seed silanization agent (sample n-ZSM-5), the spectrum shows a main peak around $1.2-1.4$ ppm corresponding to the alkyl protons in the $\mathrm{TPA}^{+}$molecule and a second peak at $5.5 \mathrm{ppm}$ assigned to water molecules. In addition, a very small peak at $11 \mathrm{ppm}$ is observed corresponding to hydrogen bonded protons [21]. The hierarchical ZSM-5 sample (h-ZSM-5) display another broad double peak at $6-8 \mathrm{ppm}$ arising from aromatic protons of the seed silanization moiety as 
well as a shoulder around 3 - 4 ppm assigned to $\mathrm{Si}-\mathrm{O} \underline{\mathrm{H}}$ or $\mathrm{Si}-\mathrm{OC} \underline{\mathrm{H}}_{n}$ - protons from surface silanols or non-completely hydrolyzed silanization agent. For the hierarchical ZSM-5 samples prepared using ethanol and n-butanol as alcohols in the silanization step, this peak becomes more intense, confirming the presence of alkoxy species anchored to the surface of these samples. This shoulder is especially marked for the h-ZSM-5 (n-but) sample suggesting the incorporation of a significant number of n-butoxy moieties on the surface of the proteozeolitic units, which remain anchored even after the hydrothermal treatment. This fact could be the cause of the X-ray amorphous nature of this sample. Seemingly, a high degree of alkoxylation of the protozeolitic units may strongly hinder the nanounit aggregation, precluding the zeolite crystallization. Figure 7 is a schematic representation of the incorporation of both PHAPTMS and alkoxy moieties during the silanization step on the external surface of the proteozeolitic units (represented herein with SDA molecules embedded within the pore structure). It must be noted that, although in this scheme the nanounits are shown as having a perfect a MFI structure, the real picture is probably quite more complex. This point is still the subject of intense debate in the literature with some authors proposing that the nanounits formed in the early stages of ZSM-5 crystallization are perfectly crystalline, whereas others supports they are completely amorphous and that they do not have any participation in the subsequent zeolite crystallization. A third option, which we consider as more feasible, is that these nanounits possess some kind of organization, being formed by aluminosilicate species interacting and surrounding the TPA ${ }^{+}$molecules. In this way, the protozeolitic nanounits could not be considered as completely amorphous material, although they are far from having a perfect MFI structure. According to this proposal, and based on our results, the protozeolitic nanounits are transformed into perfect zeolite entities during the hydrothermal treatment, which seems to be always linked to their aggregation into larger particles. 
The presence of alkoxy groups in the samples has been also probed by the solid-state ${ }^{13} \mathrm{C}$ CP-MAS NMR spectra (see Figure 8). For the reference $\mathrm{n}-\mathrm{ZSM}-5$ sample, ${ }^{13} \mathrm{C}$ signals at 10.0 and $11.0 \mathrm{ppm}, 16 \mathrm{ppm}$, and $63 \mathrm{ppm}$ are resolved from distinct carbon moieties associated with the TPA+ propyl chains. These signals are also present in the ${ }^{13} \mathrm{C}$ CP-MAS spectrum of the hierarchical h-ZSM-5 sample prepared from silanized seeds, along with ${ }^{13} \mathrm{C}$ signals at 23,47 , 113,129 , and $149 \mathrm{ppm}$ from the PHAPTMS species. In this case, the lower and broader relative signal intensities associated with PHAPTMS species, as compared to TPA ${ }^{+}$, are consistent with their less ordered environments, as the former are located on the outer part of zeolite nanounits [21]. Hierarchical samples, silanized in the presence of alcohols, show signals at the same chemical shifts as h-ZSM-5 sample, with the broadening of the signal at $63 \mathrm{ppm}$ attributed to the $\mathrm{Si}-\underline{\mathrm{C}}_{\mathrm{n}}$ - carbon, which it is probably due to the alcohol grafting via alkoxylation, especially for the samples silanized in the presence of ethanol and $\mathrm{n}$-butanol. Interestingly, the ${ }^{13} \mathrm{C}$ signals corresponding to the silanization agent are clearly more intense in the samples prepared adding ethanol and n-butanol to the silanization step. This finding can be interpreted as a consequence of the cross-polarization technique used for the acquisition of the NMR signal, where the magnetization of the ${ }^{13} \mathrm{C}$ nuclei is achieved through the neighboring ${ }^{1} \mathrm{H}$. This indicates that PHAPTMS species in samples h-ZSM-5 (eth) and h-ZMS-5 (n-but) are surrounded by a larger number of protons compared to the other samples. Therefore, this result is a clear evidence of the surface grafting of protic species such as ethoxy or n-butoxy groups on the silica surface in close vicinity to the silanization moieties (Figure 7). Moreover, it indicates that in the case of ethanol, and especially of n-butanol, the alkoxy moieties interact strongly with the adjacent organosilane species, which is caused by both types of species being formed by linear molecules, which favors their alignment in parallel directions and their lateral interaction. Accordingly, for $n$-butanol an organic layer is formed over the external surface of the protozeolitic units consisting in both oganosilane and butoxy groups. This layer is expected to be quite stable 
and hydrophobic, which results in avoiding almost completely the aggregation of the nanounits and, therefore, in hindering the appearance of crystallinity. In the case of ethanol, this effect is less pronounced as the ethoxy group is less hydrophobic and has a shorter length, which means that its interaction with the organosilane molecules is not so strong. Therefore, the layer formed with ethanol is not so stable against condensation reactions between adjacent nanounits, which in turn causes a partial and delayed growth by aggregation, leading to a material formed by a mixture of phases. Finally, when using 2-propanol and methanol as silanization solvents, the alkoxy groups formed on the external surface of the protozeolitic units seem to be the least stable due to a combination of factors: lower size, non-linear configuration and lower hydrophobicity. As a consequence, isopropoxy and methoxy groups can be easily removed from the external surface during the final hydrothermal treatment, i.e. they do not hinder the nanounit aggregation, leading to highly crystalline ZSM-5 samples with hierarchical porosity.

Finally, in order to test the catalytic properties of the different samples they were checked in the catalytic cracking of LDPE at $340^{\circ} \mathrm{C}$ using a plastic / catalyst mass ratio of 100 . The results obtained are summarized in Table 2. All the samples gave rise to conversions clearly higher than the thermal cracking $(1.2 \%)$, with values varying within $18-56 \%$. However, the conversions cannot be compared directly among the samples due to their different aluminum content (see Table 1). In order to perform a better comparison among them, the activity was expressed in terms of TOF ( $\mathrm{g}$ plastic converted) ( $\mathrm{g}$ of $\mathrm{Al})^{-1} \mathrm{~s}^{-1}$. This parameter allows normalizing the effect of the aluminum content. The TOF values of the different samples are compared in Figure 9. It can be seen that some of the hierarchical samples synthesized under the presence of alcohols (hZSM-5 (2-prop) and h-ZSM-5 (meth)) show even better TOF values than the reference n-ZSM-5 and h-ZSM-5 materials. It must be noted that the occurrence of zeolite crystallinity is compulsory for the catalytic cracking to occur at temperatures as low as $340^{\circ} \mathrm{C}$, since amorphous materials (e.g. Al-MCM-41 and amorphous silica-alumina) are practically inactive under these conditions. 
Consequently, the high TOF values of these two samples are caused by their total zeolite crystallinity, as well as by the improved accessibility and diffusion of the bulky plastic macromolecules, caused by their enhanced secondary porosity in terms of both surface area and pore volume. On the other hand, the other two samples (h-ZSM-5 (eth) and h-ZSM-5 (n-but)) presented worse TOF values than the two reference materials, which can be related to the noncompletely crystalline nature of these samples. However, it is remarkable that in the case of the material prepared with $n$-butanol, its catalytic activity is far from being negligible in spite of being X-ray amorphous. This result can be considered as an indication that the nanounits in this sample present some kind of zeolite-like organization, as they are really intermediate species in the zeolite crystallization process.

\section{Conclusions}

The addition of alcohols along with the silanization agent in the synthesis of hierarchical ZSM-5 by the seed silanization method affects meaningfully to the properties of the final materials. Two effects can be envisaged to explain the sharp variations observed with the addition of alcohols in the silanization step: Firstly, an increase in the incorporation degree of the silanization agent due to the reduction of the gel viscosity caused by the alcohols, which favors the interaction and grafting of the organosilane with the protozeolitic nanounits. Secondly, alkoxylation reactions also occur by anchoring of the alcohols on the external surface of the protozeolitic units. Accordingly, an organic layer is formed around the latter consisting in both oganosilane and alkoxy groups. Depending on the employed alcohol, this layer is more or less stable and hydrophobic, which in some cases results in avoiding almost completely the aggregation of the nanounits and, therefore, in hindering the appearance of crystallinity even after an intense hydrothermal treatment. For n-butanol this effect is specially pronounced due to the linear and hydrophobic nature of this alcohol, which promotes the interaction with adjacent 
organosilane species over the nanounit external surface, leading to a strong protection against aggregation, which in this system implies hindering the zeolite crystallization process.

In the case of the addition of 2-propanol and methanol the protective organic layer formed around the nanounits is not so stable, which allows highly crystalline samples to be produced. Moreover, as the alcohol increases the organosilane incorporation, the hierarchical features of the ZSM-5 so obtained are clearly enhanced compared to the reference h-ZSM-5 sample, leading to a further improvement of their textural properties, accessibility and catalytic activity in the conversion of bulky compounds, as it is the case of the LDPE cracking reaction here employed as catalytic test.

\section{Acknowledgements}

The authors thank the financial support from "Secretaría General de Política Científica y Tecnológica, Ministerio de Educación y Ciencia” in Spain (project ENE2008-05471). 


\section{References}

[1] M. Hartmann, Angew. Chem. Int. Ed. 43 (2004) 5880

[2] K. Egeblad, C. H. Christensen, M. Kustova, C. H. Christensen, Chem Mater. 20 (2008) 946

[3] D. P. Serrano, J. Aguado, J. M. Escola, J. M. Rodríguez, A. Peral, Chem. Mater. 18 (2006) 2462

[4] C.J. H. Jacobsen, C. Madsen, J. Houzvicka, J. Schmidt, A. Carlsson, J. Am. Chem. Soc. 122 (2000) 7116 .

[5] J. C. Groen, L. A. A. Peffer, J. A. Moulijn, J. Perez Ramirez, Chem Eur. J. 11 (2005) 4983.

[6] M. Ogura, S. Shinomiya, J. Tateno, Y. Nara, E. Kikuchi, M. Matsukata, Chem. Lett. (2000) 882.

[7] J. Perez Ramirez, D. Verboekend, A. Bonilla, S. Abelló, Adv. Funct. Mater. 19 (2009) 3972.

[8] F. S. Xiao, L. Wang, C. Yin, K. Lin, Y. Di, J. Li, R. Xu, D. S. Su, R. Schlogl, T. Yokoi, T. Tatsumi, Angew. Chem. Int. Ed. 45 (2006) 3090.

[9] M. Choi, H. S. Cho, R. Srivastava, C. Venkatesan, D. H. Choi, R. Ryoo, Nature Mater. 5 (2006) 718.

[10] H. Wang, T. J. Pinnavaia, Angew. Chem. Int. Ed. 45 (2006) 7603.

[11] C. H. Christensen, K. Johansen, I. Schmidt, C. H. Christensen, J. Am. Chem. Soc. 125 (2003) 13370.

[12] C. H. Christensen, K. Johansen, E. Tornqvist, I. Schmidt, H. Topsoe, C. H. Christensen, Catal. Today 128 (2007) 117.

[13] J. C. Groen, W. Zhu, Browuer, S. T. Huynink, F. Kapteijn, J. A. Moulijn, J. Perez Ramirez, J. Am. Chem. Soc. 129 (2007) 355.

[14] R. Srivastava, M. Choi, R. Ryoo, Chem. Commun. (2006) 4489.

[15] J. Kim, M. Choi, R. Ryoo, J. Catal. 269 (2010) 219.

[16] D. H. Lee, M. Choi, B. W. Yu, R. Ryoo, Chem. Commun. (2009) 74. 
[17] C. H. Christensen, I. Schmidt, A. Carlsson, K. Johansen, K. Herbst, J. Am. Chem. Soc. 127 (2005) 8098.

[18] A. H. Janssen, A. J. Koster, K. P. de Jong, Angew. Chem. Int. Ed. 40(6) (2001) 1103.

[19] R. Szostak, Stud. Surf. Sci. Catal. 137 (2001) 261.

[20] J. C. Groen, J. C. Jansen, J. A. Moulijn, J. Perez Ramirez, J. Phys. Chem. B 108 (2004) 13062.

[21] D. P. Serrano, J. Aguado, G. Morales, J. M. Rodriguez, A. Peral, M. Thommes, J. D. Epping, B. F. Chmelka, Chem. Mater. 21 (2009) 641

[22] J. Aguado, D. P. Serrano, J. M. Escola, A. Peral, J. Anal. Appl. Pyrol. 85 (2009) 352.

[23] J. Aguado, D. P. Serrano, J. M. Rodriguez, Micropor. Mesopor. Mater. 115 (2008) 504.

[24] K. Egeblad, C. H. Christensen, M. Kutsova, C. H. Christensen, Chem. Mater. 20 (2008) 946.

[25] R. Chal, C. Gerardin, M. Bulut, S. Van Donk, Chem. Cat. Chem., DOI:

10.1002/cctc.201000158.

[26] D. P. Serrano, R. Sanz, P. Pizarro, I. Moreno, Chem. Commun. 11 (2009) 1407.

[27] D. P. Serrano, J. Aguado, J. M. Escola, J. M. Rodriguez, A. Peral, J. Mat. Chem. 18 (2008) 4210.

[28] J. M. Fedeyko, D. G. Vlachos, R. F. Lobo, Micropor. Mesopor. Mater. 90 (2006) 102.

[29] D. T. On, S. Kaliaguine, Angew. Chem. Int. Ed.40(17) ( 2001) 3248.

[30] G. Coudurier, C. Naccache, J. C. Vedrine, J. Chem. Soc., Chem. Commun. (1982) 1413. 
Table 1. Textural properties (Ar adsorption measurements), Si/Al ratio (ICP-AES) and optical density ratio (ODR, from FITIR spectra) of ZSM-5 materials prepared following different silanization and hydrothermal synthesis treatments.

\begin{tabular}{|c|c|c|c|c|c|c|c|c|}
\hline Sample & $\begin{array}{c}\text { ODR } \\
\left(\left.I_{550}\right|_{450}\right.\end{array}$ & Si/AI & $\begin{array}{c}S_{\text {BET }} \\
\left(\mathrm{m}^{2} / \mathrm{g}\right)\end{array}$ & $\begin{array}{c}S_{Z M P} \\
\left(\mathrm{~m}^{2} / \mathrm{g}\right)\end{array}$ & $\begin{array}{c}S_{S P} \\
\left(\mathrm{~m}^{2} / \mathrm{g}\right)\end{array}$ & $\begin{array}{c}V_{\text {тот }} \\
\left(\mathrm{cm}^{3} / \mathrm{g}\right)\end{array}$ & $\begin{array}{c}V_{Z M P} \\
\left(\mathrm{~cm}^{3} / \mathrm{g}\right)\end{array}$ & $\begin{array}{c}V_{S P} \\
\left(\mathrm{~cm}^{3} / \mathrm{g}\right)\end{array}$ \\
\hline n-ZSM-5 & 0.864 & 36 & 420 & 344 & 74 & 0.403 & 0.198 & 0.205 \\
\hline h-ZSM-5 & 0.822 & 35 & 609 & 210 & 399 & 0.593 & 0.121 & 0.472 \\
\hline $\begin{array}{c}\text { h-ZSM-5 } \\
\text { (2-prop) }\end{array}$ & 0.792 & 41 & 719 & 267 & 452 & 0.675 & 0.154 & 0.521 \\
\hline $\begin{array}{c}\text { h-ZSM-5 } \\
\text { (meth) }\end{array}$ & 0.748 & 33 & 699 & 180 & 519 & 0.573 & 0.104 & 0.469 \\
\hline $\begin{array}{c}\text { h-ZSM-5 } \\
\text { (eth) }\end{array}$ & 0.726 & 37 & 729 & 100 & 629 & 1.282 & 0.055 & 1.227 \\
\hline $\begin{array}{c}\text { h-ZSM-5 } \\
\text { (n-but) }\end{array}$ & 0.492 & 35 & 585 & 28 & 557 & 1.612 & 0.016 & 1.596 \\
\hline
\end{tabular}

SzMP: micropore surface area; SsP: secondary porosity surface area

$V_{\text {ZMP: }}$ micropore volume; $V_{S P}$ : secondary porosity volume 
Table 2. Cracking of LDPE over different ZSM- 5 zeolite catalysts $\left(T=340^{\circ} \mathrm{C} ; \mathrm{LDPE} /\right.$ catalyst $=$ $100 \mathrm{w} / \mathrm{w} ;$ time $=2 \mathrm{~h}$ ).

\begin{tabular}{|l|c|c|c|c|}
\hline \multirow{2}{*}{ Sample } & Conversion & \multicolumn{3}{|c|}{ Selectivity by groups (wt\%) } \\
\cline { 3 - 5 } & $(\%)$ & $\mathbf{C}_{1}-\mathbf{C}_{5}$ & $\mathbf{C}_{6}-\mathbf{C}_{12}$ & $\mathbf{>}_{\mathbf{C}_{13}}$ \\
\hline None & 1.2 & 100.0 & 0.0 & 0.0 \\
\hline n-ZSM-5 & 27.4 & 66.1 & 33.8 & 0.1 \\
\hline h-ZSM-5 & 44.1 & 67.3 & 32.7 & 0.0 \\
\hline h-ZSM-5 (2-prop) & 56.2 & 59.8 & 40.0 & 0.2 \\
\hline h-ZSM-5 (meth) & 56.7 & 66.8 & 33.2 & 0.0 \\
\hline h-ZSM-5 (eth) & 26.3 & 77.7 & 22.1 & 0.2 \\
\hline h-ZSM-5 (n-but) & 18.5 & 92.3 & 7.5 & 0.1 \\
\hline
\end{tabular}




\section{Figure and Scheme captions}

Figure 1. XRD patterns $(A)$ and FTIR spectra $(B)$ of the as-synthesized nanocrystalline n-ZSM-5, and the hierarchical h-ZSM-5 zeolites.

Figure 2. Cumulative pore volume and pore size distribution, obtained from NLDFT calculations of Ar adsorption-desorption isotherms at $87.4 \mathrm{~K}$, of calcined n-ZSM-5 and hierarchical h-ZSM-5 zeolites.

Figure 3. TG and DTG of the as synthesized n-HZSM-5 (A), h-ZSM-5 (B) and h-ZSM-5 (eth) (C) samples.

Figure 4. TEM micrographs of calcined $n-Z S M-5$ and $h-Z S M-5$ reference samples.

Figure 5. TEM micrographs of calcined hierarchical ZSM-5 samples.

Figure 6. Solid-state ${ }^{1} \mathrm{H}$ MAS NMR spectra of the as-synthesized n-ZSM-5 and hierarchical ZSM5 samples.

Figure 7. Schematic diagram showing the distribution of PHAPTMS on the surface of proteozeolitic units after the silanization step (A), the incorporation of alkoxy moieties by alkoxylation when the silanization proceeds in the presence of alcohols $(B)$ and the interaction between PHAPTMS and n-butoxy grafted species in close vicinity on the surface of proteozeolitic units $(C)$.

Figure 8. Solid-state ${ }^{13} \mathrm{C}$ CP-MAS NMR spectra of the as-synthesized n-ZSM-5 and hierarchical ZSM-5 samples.

Figure 9. TOF values obtained with the different samples in the catalytic cracking of LDPE at $340^{\circ} \mathrm{C}$. 
Figure 1
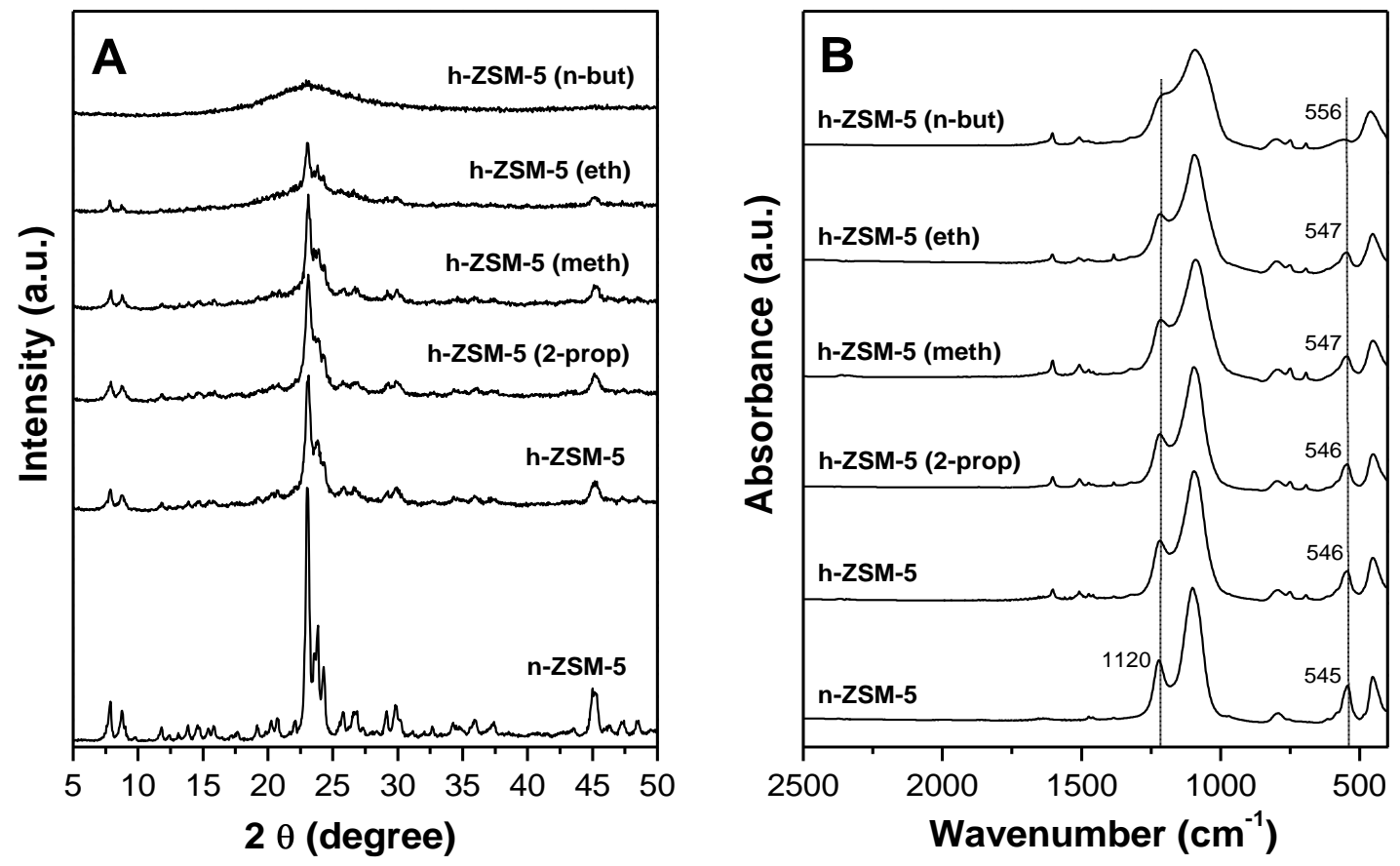
Figure 2

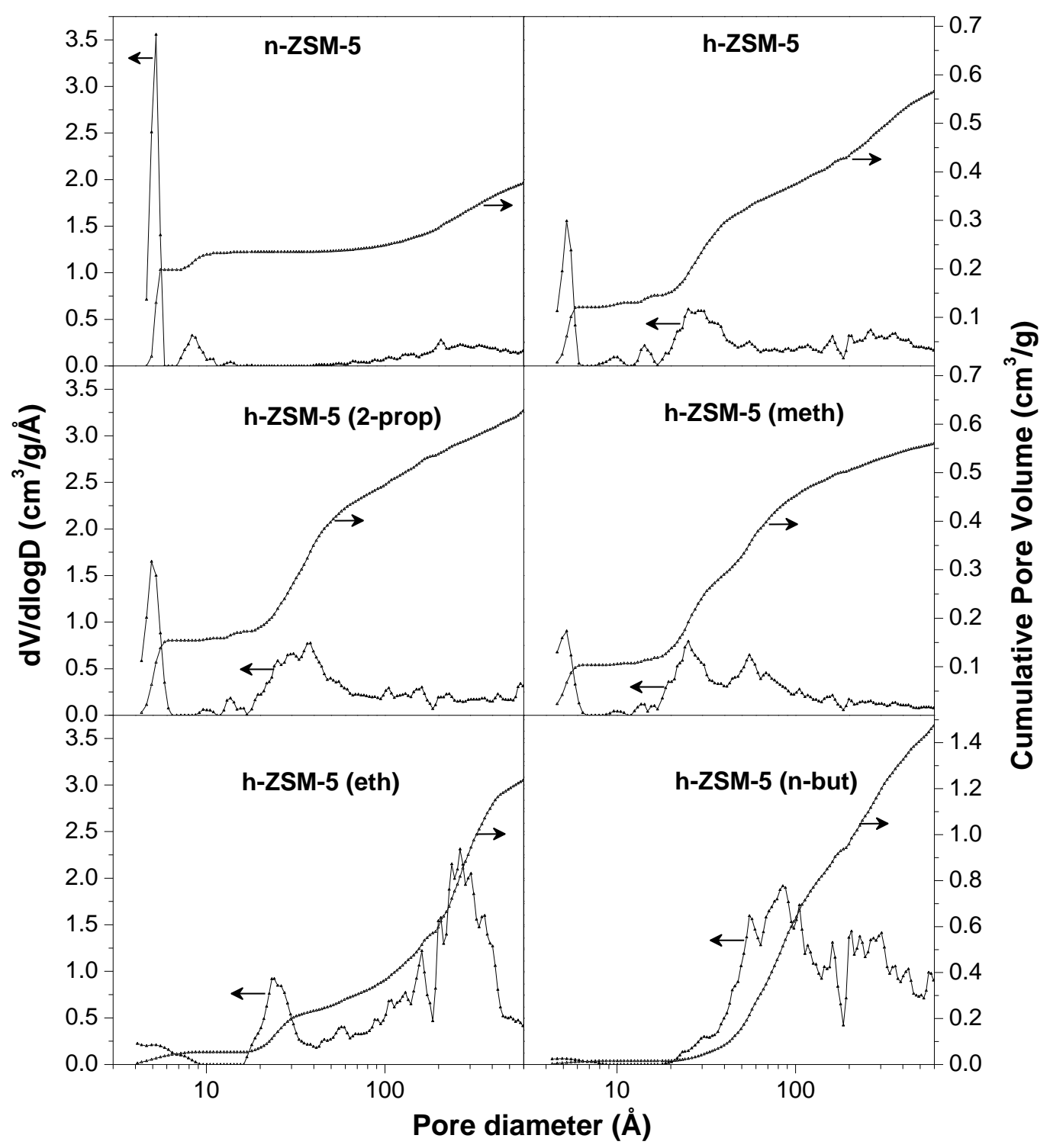


Figure 3

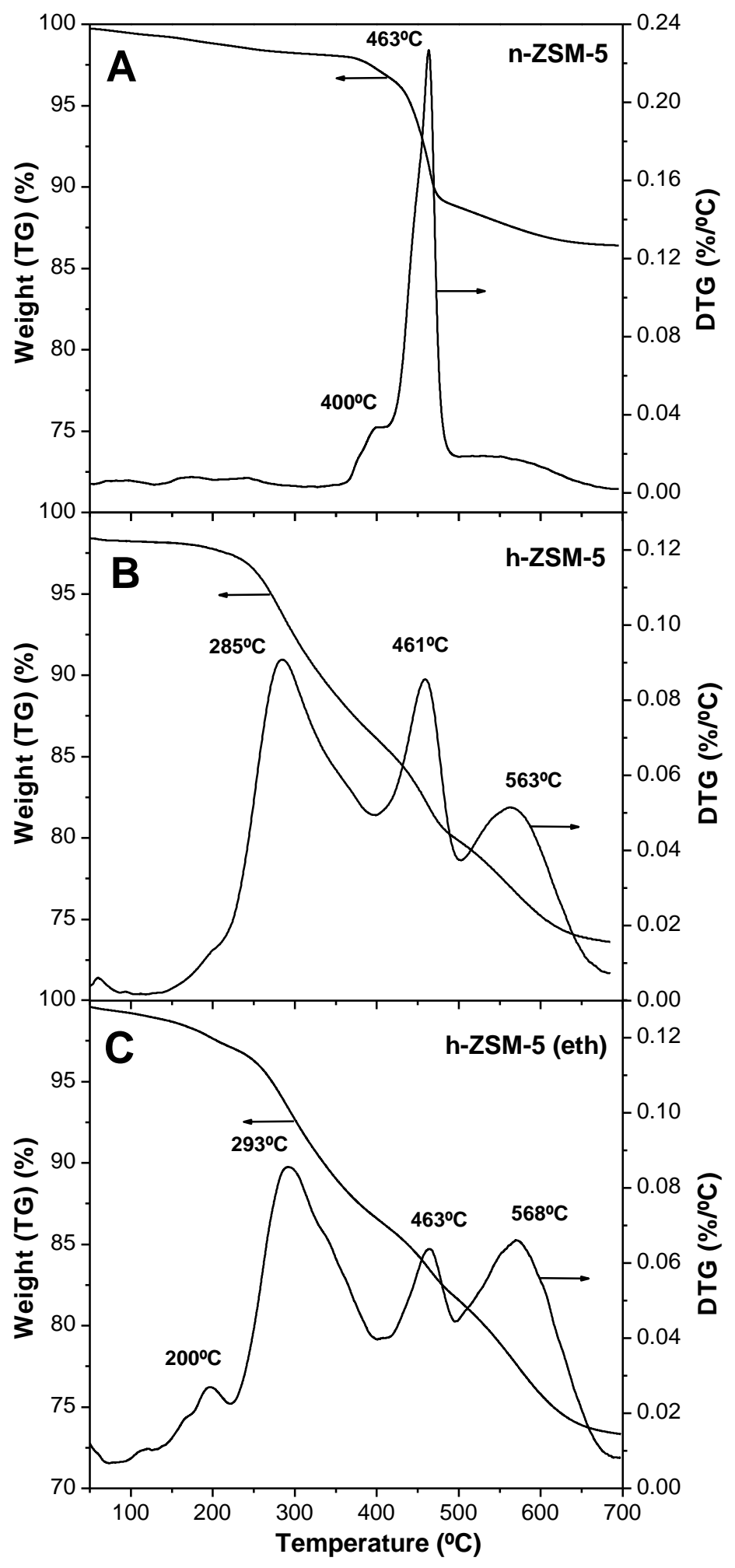


Figure 4
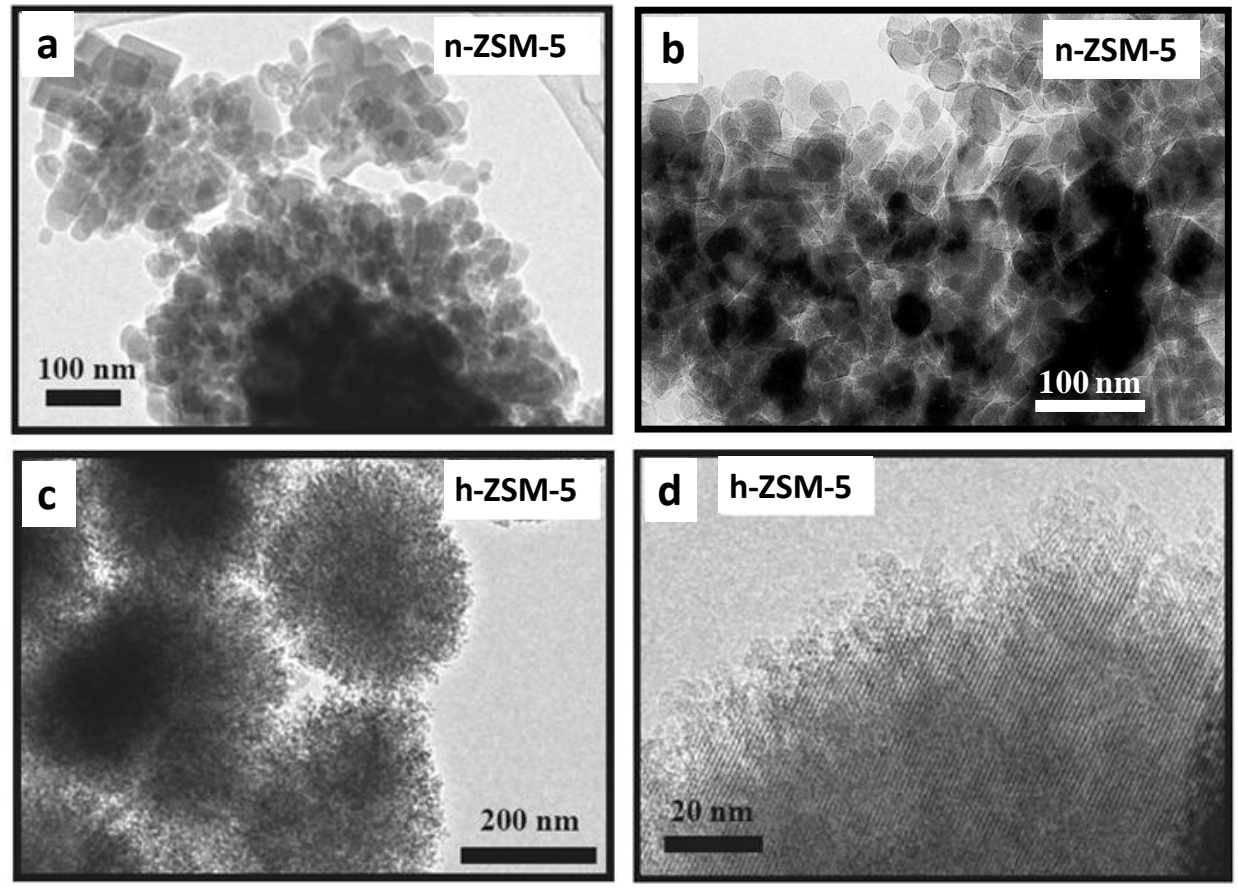
Figure 5
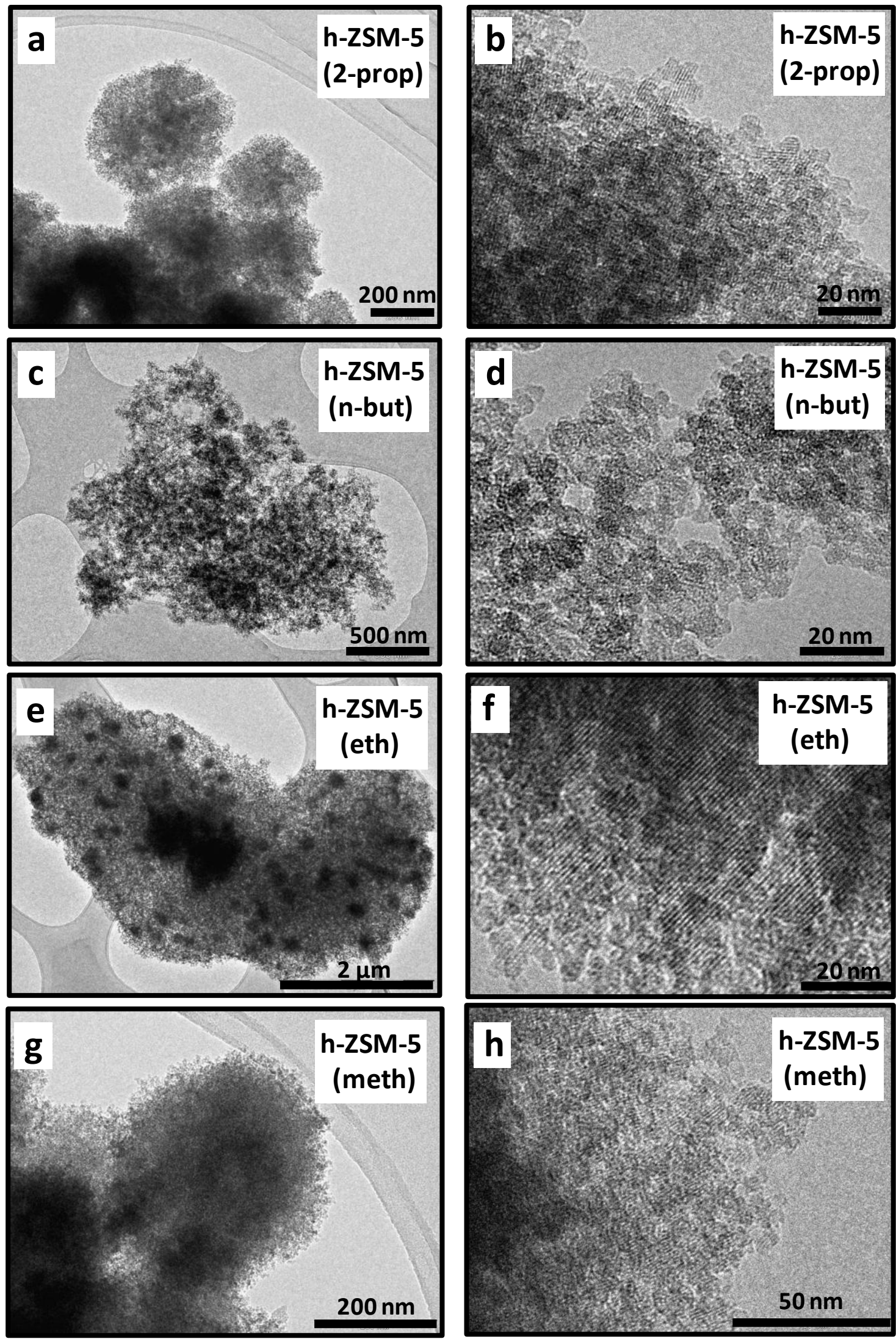
Figure 6

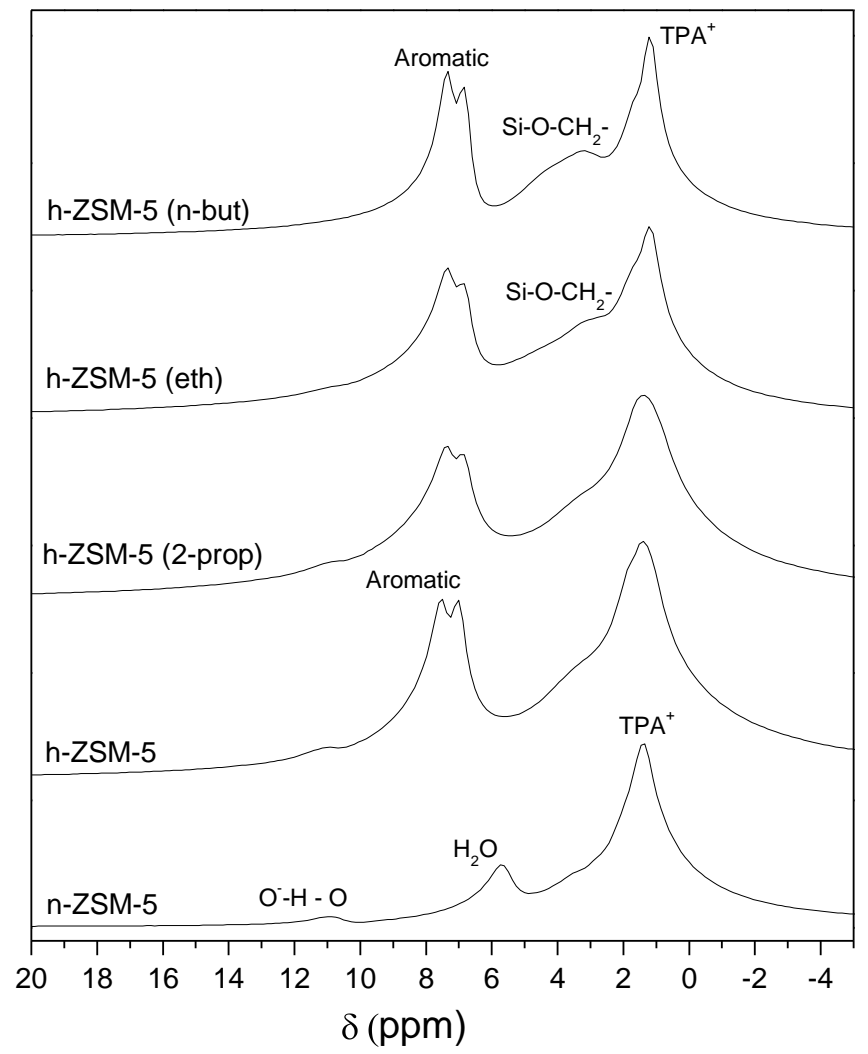


Figure 7

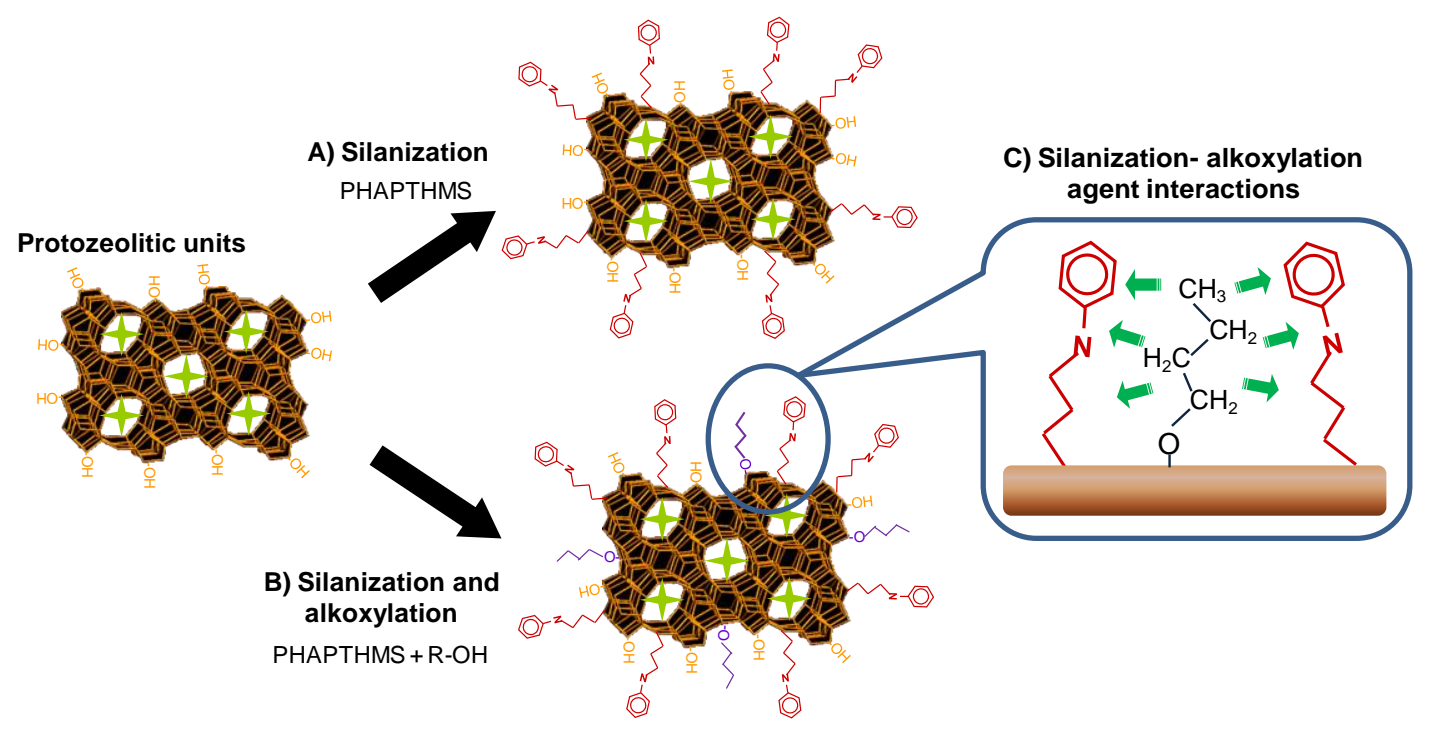




\section{Figure 8}

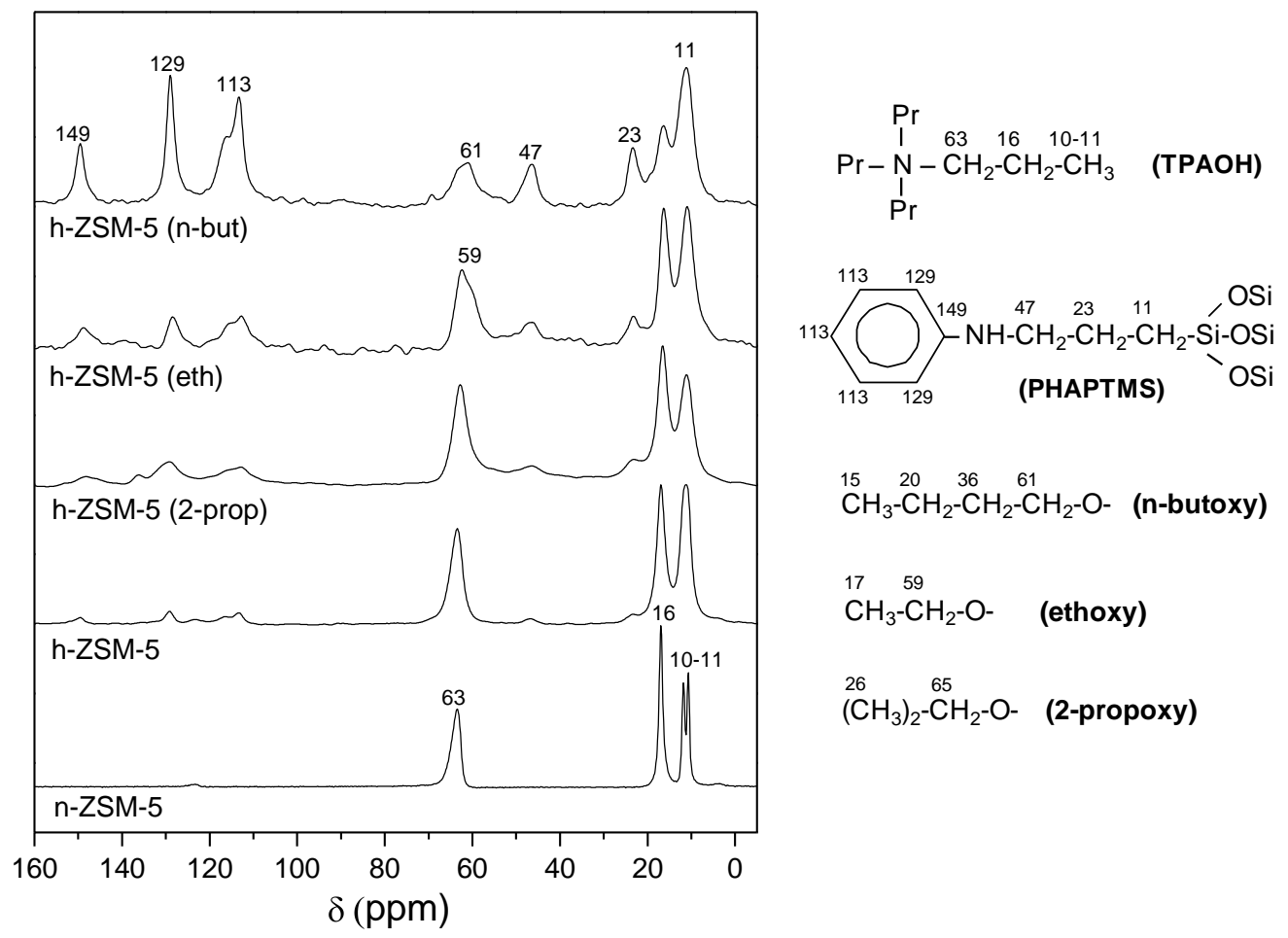


Figure 9

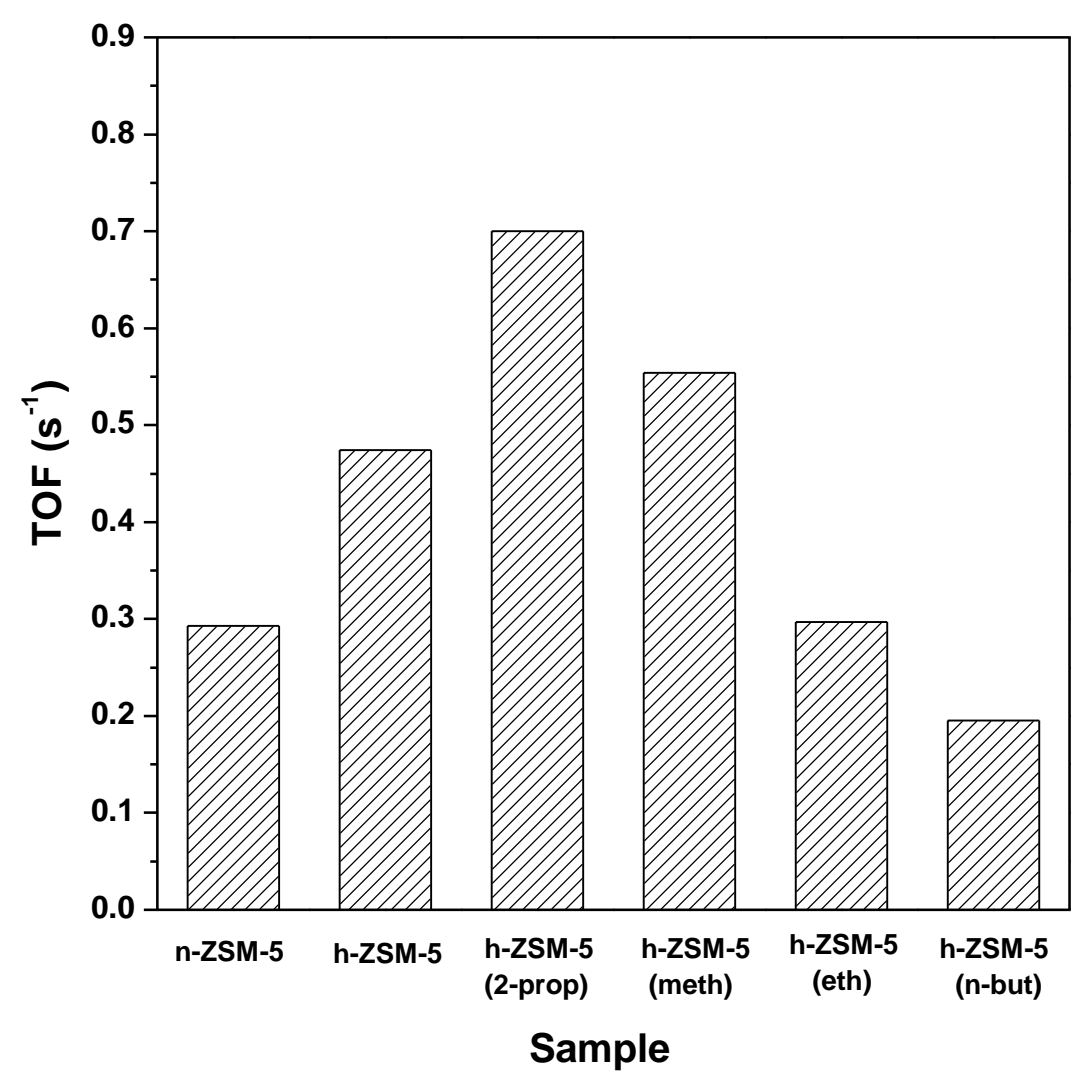

